\title{
ADHERENCIA TERAPÉUTICAY FUNCIONALIDAD FAMILIAR EN PERSONAS CON ARTRITIS REUMATOIDE
}

\author{
ADHERENCE AND FAMILY \\ FUNCTIONING IN PEOPLE WITH \\ RHEUMATOID ARTHRITIS \\ Recibido 30 de abril 2015 \\ Aceptado 1 de mayo 2015
}

Correspondencia: Mtra. María Elena Alberto Bazán Calle 5 No. 88 Privada 5 Colonia Agrícola Pantitlán Delegación Iztacalco, C.P. 08100 Tel. 5515512754, celular 0445531063361 maria.albert@hotmail.es

\section{Autores:}

Mtra. María Elena Alberto Bazán

Profesora de Tiempo Competo de la FES Zaragoza, UNAM. Carrera de Enfermería. Conjuntamente adscrita al Hospital General la Perla, ISEM. Responsable de la Investigación

Mtra. Olga Taboada Aranza

Profesora de Tiempo Competo de la FES Zaragoza, UNAM. Carrera de Odontología

Mtro. Gerardo Reyes Hernández

Profesor de Tiempo Competo de la FES Zaragoza, UNAM. Carrera de Psicología

Palabras clave: Adherencia Terapéutica, Funcionalidad Familiar y Arritis Reumatoide

Keywords: Adhesion Therapeutics, Family Functionality and Rheumatoid Arthritis 


\section{RESUMEN}

La Artritis Reumatoide (AR) se ha incrementado de manera alarmante, el Instituto Nacional de Artritis y Enfermedades Musculoesqueléticas y de la Piel en los EU, reporta que 1:10 habitantes la desarrolla; en México un millón 700 mil personas entre 20 y 50 años la padecen, lo que coincide con la edad productiva, constituye un grave problema de Salud Pública su costo anual es de 600 dólares por paciente, la incidencia es mayor en las mujeres con respecto a los hombres 3:1; el panorama se agudiza ante ausencias laborales recurrentes por discapacidad debido a la presencia de dolor, deformidad, rigidez e inflamación articular y la disminución de la fuerza muscular, suscitando impacto económico y emocional para el individuo afectado así como para los integrantes de la familia, impulsándolos a reorganizarse y en la mayoría a desintegrarse como lo estableció Virginia Satir; la baja Adherencia Terapéutica (AT) condiciona resultados desfavorables y gastos innecesarios para el sector salud. Es necesario identificar la adherencia terapéutica y la funcionalidad familiar en las personas con AR, se realizó un estudio observacional, prolectivo, descriptivo y transversal; la población se conformó por 100 pacientes, usuarios del Hospital General Ignacio Zaragoza, Instituto de Seguridad y Servicios Sociales de los Trabajadores del Estado.(ISSSTE); con una media de edad de 47 ( \pm 10.7$), 74$ mujeres y 26 hombres, con un mes de tratamiento antirreumático. Al análisis por sexo se observa mayor frecuencia de mujeres $(n=38)$ en pertenecer a una familia conflictiva y baja AT, mientras los hombres ( $n=22)$ ubicados en familias nutricias en mayor proporción y mediana AT; revisando la AT por etapas de la vida, se descubre que el adulto joven y el maduro ocupan 30 y 32 casos respectivamente, con baja y mediana AT; este dato es importante si la esperanza de vida en México es de 80 años; cabría preguntarse qué calidad de vida le espera a estos pacientes.

Palabras clave: Felicidad, estudiantes de enfermería y medicina, empoderamiento de la profesión

\section{ABSTRACT}

Rheumatoid Arthritis (RA) has increased alarmingly, the National Institute of Arthritis and Musculoskeletal and Skin Diseases in the US, reports that develops 1:10 inhabitants; in Mexico one million 700 thousand people between 20 and 50 years suffer, which coincides with the productive age, is a serious public health problem annual cost is \$ 600 per patient, the incidence is higher in women compared to men 3: 1; the outlook worsens with recurrent absenteeism disability due to the presence of pain, deformity, stiffness and joint swelling and decreased muscle strength, raising economic and emotional impact for the affected individual and for family members, urging to reorganize and most established to disintegrate as Virginia Satir; Low Adherence Therapy (AT) conditions unfavorable results and unnecessary costs to the health sector. You need to identify adherence and family functioning in people with RA, an observational, prolective, descriptive, cross-sectional study; the population was formed by 100 patients, users of General Ignacio Zaragoza Hospital, ISSSTE; with a mean age of 47 ( \pm 10.7), 74 women and 26 men, with a month antirheumatic treatment. Analysis by sex more often in women $(n=38)$ belong to a family conflict and low AT is observed, while men ( $n=22)$ located in nurturing families in greater proportion and medium AT: AT reviewing the stages of life, discovers that the young adult and mature occupy 30 and 32 cases respectively, low and medium AT; this is important if life expectancy in Mexico is 80 years; one might ask what quality of life awaits these patients.

Keywords: Adhesion Therapeutics, Family Functionality and Rheumatoid Arthritis.

\footnotetext{
140 4MN Why Vol.14. No. 3 septiembre - diciembre 2015
} 


\section{INTRODUCCIÓN}

a adherencia al tratamiento o cumplimiento terapéutico se ha definido como el contexto en el cual el comportamiento de la persona coincide con las recomendaciones relacionadas con la salud, sin embargo la repercusión de la poca o nula adherencia terapéutica crece a medida que se incrementa la carga de enfermedad crónica, lo cual represento el 54\% en el 2001, considerando lo anterior se estima un 65\% de no adherencia para el 2020'. Estas referencias numéricas son alarmantes lo que manifiesta la necesidad de trabajar arduamente en la promoción de la adherencia a los tratamientos de largo plazo. Existen estudios en países desarrollados que exponen que la adherencia a los tratamientos prolongados en la población general es del 50\%, siendo menor en los países en desarrollo?2.

La Organización Mundial de la Salud (OMS) asegura que el incumplimiento del tratamiento es la principal causa de que no se obtengan todos los beneficios que los medicamentos pueden proporcionar a los pacientes. Razón por la cual se manifiestan consecuencias como descompensación de los síntomas, complicaciones físicas, psicosociales así como los reingresos hospitalarios, se manifiestan bajos resultados de salud y el aumento significativo de costos, en ese sentido la población es afectada a partir de la perspectiva de la calidad de vida y por supuesto en la economía de la salud

Ante las cifras actuales de pacientes crónicos en continuo aumento, la enfermera contemporánea ha comenzado a redireccionar su papel frente a estas afecciones, considerando que cuidar es una actividad dinámica, reflexiva, deliberada y basada en la interacción humana, y en el compromiso de promover el bienestar del otro como lo admite Collieré5, quien puntualiza que el cuidado es "todo lo que ayuda a vivir y permite existir" así como dirigir acciones a todo lo que estimula la vida, en consecuencia la disciplina de enfermería, debe apuntar al cuidado familiar, espacio donde el individuo afectado o no se desenvuelve diariamente, por lo que la modificación o permanencia de los hábitos adquiridos a través de la familia solo podrán ser abatidos con la incorporación de todos sus integrantes y enfatizar en la transmisión de una cultura de salud, involucrando a cada uno de sus integrantes.

Por otro lado, se ha demostrado que la adherencia terapéutica frente a la enfermedad de Artritis Reumatoide (AR), constituye un aliciente para el detenimiento de la lesión articular, cuya etiología es desconocida y el curso totalmente impredecible, con períodos de calma que se alternan con otros de exacerbación, los llamados brotes, episodios de inflamación que pueden llegar a dejar secuelas en las articulaciones afectadas y en los que el dolor se intensifica Fausett ${ }^{6}$. Además, la AR afecta no solo a la calidad de vida de quien la padece, por su naturaleza altamente incapacitante, sino también a su cantidad, ya que la esperanza de vida de estos pacientes se reduce entre cuatro y diez años Guedes, Sumont-Fischer, Leichter- Nakache y Boissier?

Al respecto la OMS considera la falta de adherencia a los tratamientos crónicos y sus consecuencias negativas clínicas y económicas un tema prioritario de salud pública ${ }^{8}$. La relación médico paciente incluye la motivación que existe en el enfermo derivada de la relación con su médico tratante, la buena comunicación y, finalmente, la confianza en la obtención de un beneficio, hace a los pacientes iniciar y mantener un tratamiento?

La presidenta de la Asociación Mexicana de Familiares y Pacientes con Artritis Reumatoide (Amepar), señala que las personas con AR son diagnosticadas tardíamente, agrego que en México no se 
cumple con la recomendación de la OMS de un reumatólogo por cada cien mil habitantes, solo se tiene el registro de 560 certificados, a proporción de 1:200,000 habitantes siendo insuficiente, incita a que las autoridades consideren a esta enfermedad como un problema de SP.

Los lazos sociales actúan no solo como soporte para mantener la salud, sino que también amortiguan los efectos negativos que sobre la salud pueden producir determinados acontecimientos de la vida. Asimismo, la falta de interés y el rechazo manifestado por las personas significativas, puede representar otro obstáculo, en su teoría Virginia Satir ${ }^{10}$ refiere que se deben conocer los sistemas de familia para determinar el grado de funcionalidad familiar, poner especial atención a los limites, jerarquía, fomento de la autoestima y los procesos de comunicación, ya que para ella una vez que el ser humano llega a este mundo, la comunicación es el único factor y el más importante que afecta la salud de una persona y sus relaciones con los demás ${ }^{11}$.

Para Satir las familias con problemas, mantienen en su círculo autoestima disminuida, comunicación indirecta, vaga y poco sincera, reglas rígidas, inhumanas, fijas e inmutables, denominando este fenómeno como familia perturbada o conflictiva, donde los cuerpos y rostros de sus integrantes manifiestan sufrimiento, cuerpos rígidos y tensos o encorvados, rostros ceñidos, tristes o indiferentes, los ojos miran hacia el suelo y más allá de la gente que está enfrente. Mientras en otro tipo de familia que reconoce, es la nutricia donde los integrantes familiares son sinceros y amorosos, tienen la libertad de comunicar lo que necesiten y pueden hablar de cualquier cosa, la autoestima individual esta fortificada, sus rostros son alegres, sus cuerpos están relajados, cuando se habla con ellos observan a la persona con atención. Una familia que estimula y aprecia las diferencias individuales, tolera los errores, donde la comunicación es abierta y las reglas de convivencia son flexibles, forma mujeres y hombres plenos ${ }^{12}$.
El termino artritis se refiere a las enfermedades que afectan a las articulaciones. Estas alteraciones pueden producir dolor articular o rigidez, daño a la estructura de una articulación o pérdida de su función. La palabra artritis proviene de las palabras griegas arthron, que significa "articulación" e itis "inflamación". Existen diversos factores para el desarrollo de la AR, por un lado tenemos el factor genético que está ligado al cromosoma 6 en el brazo corto el cual va a estar dominado con DD4, por otro lado se encuentran los factores de índole infeccioso como las mico bacterias y virus, de estos últimos los más frecuentes son el virus de la rubeola y parvovirus, con menos frecuencia se presentan los coxaquíe y los adenovirus; además se le atribuye su presencia al factor ambiental del estrés ${ }^{13}$.

El panorama implica una reflexión del profesional de enfermería a ser una pieza fundamental para redireccionar el cuidado y centrarlo en el seno familiar en virtud de incorporar programas donde la participación multidisciplinaria coloque su atención al paciente de forma individual e integral, bajo una alianza terapéutica entre el médico, la enfermera, sistema de salud, paciente y familia ${ }^{14}$, medidas que son urgentes para lograr abatir este enigma.

\section{MATERIAL Y MÉTODOS}

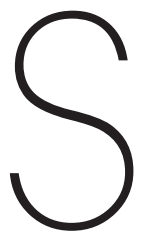
e realizó un estudio de tipo observacional, descriptivo, transversal y prolectivo.

La población de estudio estuvo conformada por 100 pacientes con diagnóstico de artritis reumatoide con un promedio de años transcurrido desde el diagnóstico de su enfermedad a la fecha con una media de 5.8 (ะ3.710), mínimo 1 máximo 16 años, siendo 74 mujeres y 26 hombres con una media de edad de 47 ( \pm 10.7), todos ellos usuarios del Hospital General Ignacio Zaragoza ISSSTE, que acudieron al servicio de consulta externa durante el periodo de agosto - octubre de 2014, con un mes de tratamiento antirreumático por lo menos y bajo consentimiento informado.

\section{2}

MNV Nhy Vol.14. No. 3 septiembre - diciembre 2015 
Se diseñó un instrumento, estructurado a partir de las variables consideradas en la presente investigación, el cual contiene en total 49 ítems, y está dividido en cuatro partes, la primera comprende datos del perfil socioeconómico del paciente(5 items), la segunda parte se consientan los datos de antecedentes de la enfermedad con (5 îtems), mientras la tercera la conforma la dimensión de la funcionalidad familiar (19 ítems) contiene los patrones de la comunicación e involucramiento familiar, resolución de problemas y autoestima; la cuarta parte involucra a la adherencia terapéutica con (19 ítems); la cual implica el conocimiento de la enfermedad, cumplimiento del tratamiento y empatía con el equipo de salud. La investigación se inició con una prueba piloto del instrumento a 50 pacientes, que cursan con enfermedad crónica degenerativa del Hospital General La Perla, observándose un grado de confiabilidad de 0.900 Kuder-Richardson.

Los datos obtenidos fueron procesados en el paquete estadístico SPSS V. 17.0 con el cual se obtuvieron las estadísticas descriptivas de las variables de estudio.

Las pruebas de significancia estadística fueron: para las variables cuantitativas la t de Student y para las variables cualitativas la prueba de KW, X2 con un nivel de confianza al 95\%. Así mismo se calculó como estimación de riesgo la razón de momios (RM) con IC95\% estableciendo como riesgo cuando la RM > 1 y el intervalo de confianza no incluyera al $1(p<0.05)$.

Cuadro 1. Sexo, tipo de familia y la determinación de la Adherencia Terapéutica

\begin{tabular}{|c|c|c|c|c|c|}
\hline \multirow[t]{2}{*}{ Variables } & \multicolumn{4}{|c|}{ Adherencia Terapéutica } & \multirow[b]{2}{*}{${ }^{*} p$} \\
\hline & BAJA & MEDIANA & RM & IC95\% & \\
\hline SEXO & & & & & \\
\hline Masculino & $00(00)$ & $26(100)$ & & & \\
\hline Femenino & $41(55.4)$ & $33(44.6)$ & 2.24 & $1.73-2.89$ & 0.0001 \\
\hline Tipo de familia & & & & & \\
\hline Nutricia & $3(6.3)$ & $45(93.8)$ & & & \\
\hline Conflictiva & $38(73.1)$ & $14(26.9)$ & 40.7 & $10.88-152.39$ & 0.0001 \\
\hline
\end{tabular}

El ser mujer representa 1.2 veces más de riesgo que el ser hombre, siendo una diferencia clínica y estadística significativa (RM=2.24; IC95\% 1.73-2.89, p<0.0001). Mientras en la familia conflictiva existe 39.7 veces más de riesgo que la familia nutricia, la diferencia es clínica y estadísticamente significativa (RM=40.7; IC95\% 10.88-152.3, p<0.0001). 
Cuadro 2. Escolaridad y la determinación de la Adherencia Terapéutica

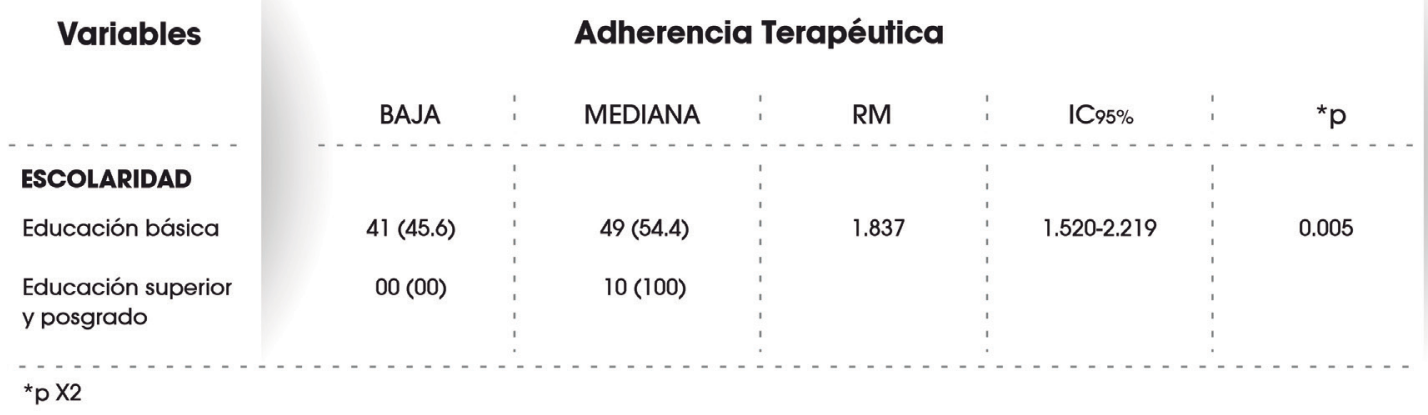

Las personas que solo cuentan con educacion basica representa una vez mas de riesgo que en aquellas personas que logran adquirir estudios de educación superior y posgrado, siendo una diferencia clínica y estadística significativa (RM=1.837; IC95\% 1.520-2.219, p<0.005).

Cuadro 3. Autoestima y la determinación de la Adherencia Terapéutica

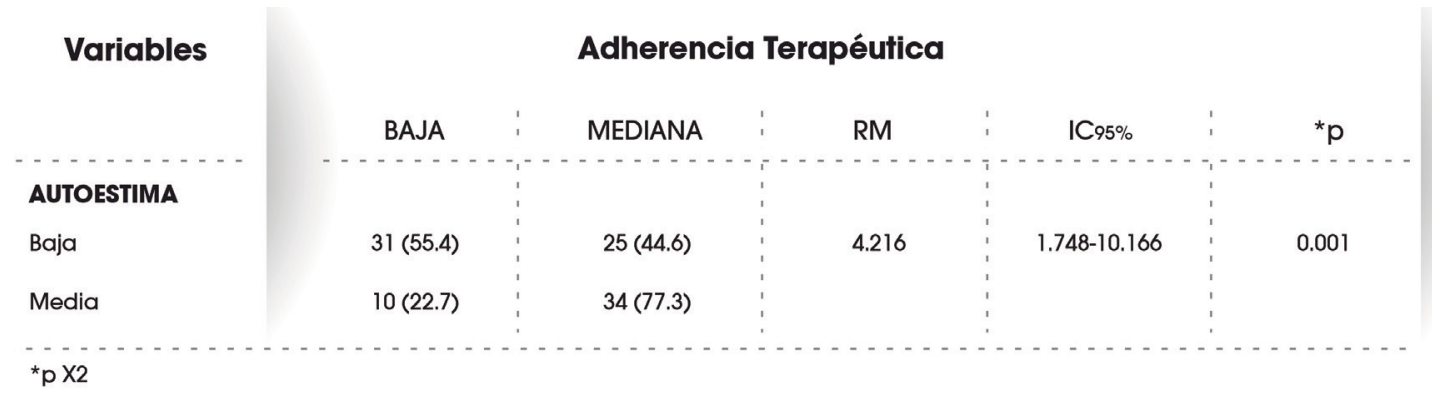

Las personas con autoestima baja representa tres veces más de riesgo que en aquellas personas que logran adquirir una autoestima media, siendo una diferencia clínica y estadística significativa (RM=4.216; IC95\% 1.748-10.166, p<0.001).

Cuadro 4. Autoestima y Tipo de Familia

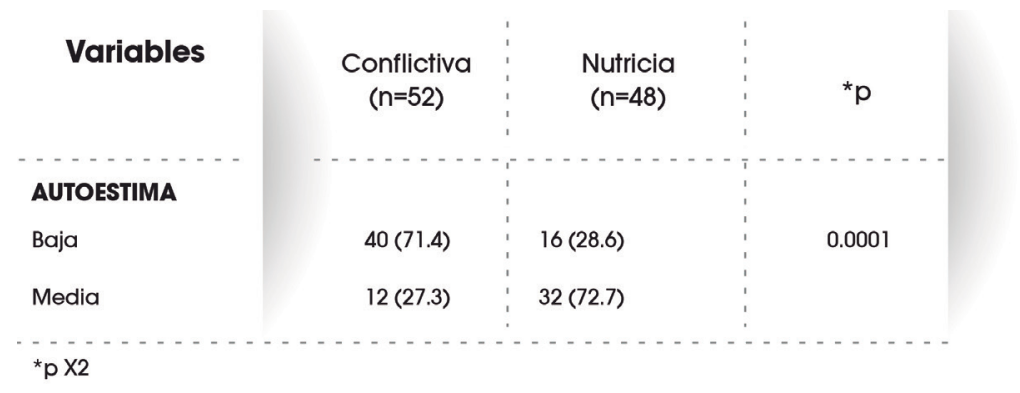

Las personas que conviven con una familia conflictiva mantienen una autoestima baja del 71.4\% y el 27.3\% logra una AT media, en cambio la familia nutricia en un 72.7\% alcanza una autoestima media, mientras 28.6\% presenta autoestima baja, siendo una diferencia estadística significativa $(p<0.0001)$. 
Cuadro 5. Conocimiento de la enfermedad y la determinación de la Adherencia Terapéutica

\begin{tabular}{|c|c|c|c|c|c|}
\hline \multirow[t]{2}{*}{ Variables } & \multicolumn{4}{|c|}{ Adherencia Terapéutica } & \multirow[b]{2}{*}{${ }^{*} p$} \\
\hline & BAJA & MEDIANA & $\mathrm{RM}$ & IC $95 \%$ & \\
\hline CONOCIMIENTO & & & & & \\
\hline Bajo & $41(44.6)$ & $51(55.4)$ & 1.804 & $1.502-2.167$ & 0.020 \\
\hline Alto & $00(00)$ & $8(100)$ & & & \\
\hline
\end{tabular}

Las personas con conocimiento bajo de la enfermedad representa una vez más de riesgo que en aquellas personas que logran un alto conocimiento de su padecimiento, siendo una diferencia clínica y estadística significativa (RM=1.804; IC95\% 1.502-2.167, p<0.020).

Cuadro 6. Etapas de la vida y la determinación de la Adherencia Terapéutica

\begin{tabular}{|c|c|c|c|c|c|}
\hline \multirow[t]{2}{*}{ Variables } & \multicolumn{4}{|c|}{ Adherencia Terapéutica } & \multirow[b]{2}{*}{${ }^{*} p$} \\
\hline & BAJA & MEDIANA & RM & $\mathrm{IC} 95 \%$ & \\
\hline CONOCIMIENTO & & & & & \\
\hline $\begin{array}{l}\text { Adulto joven y } \\
\text { maduro }\end{array}$ & $32(42.7)$ & $43(57.3)$ & 1.323 & $0.539-3.374$ & 0.642 \\
\hline Adulto mayor & $9(36.0)$ & $16(64.0)$ & & & \\
\hline
\end{tabular}

Los adultos jóvenes y maduros representa una vez más de riesgo que en los adultos, mayores siendo una diferencia clínica significativa, pero no estadísticamente significativa (p>0.0642). Se observa qua el 75 de los casos pertenecen a la etapa de la vida de adulto joven y maduro los cuales solo alcanzan la baja y mediana AT, éste dato es importante considerando que la esperanza de vida en México es de aproximadamente 80 años, por lo cual cabría preguntarse qué calidad de vida les espera a estos pacientes.

Cuadro 7. Empatía con el equipo de salud y la determinación de la Adherencia Terapéutica

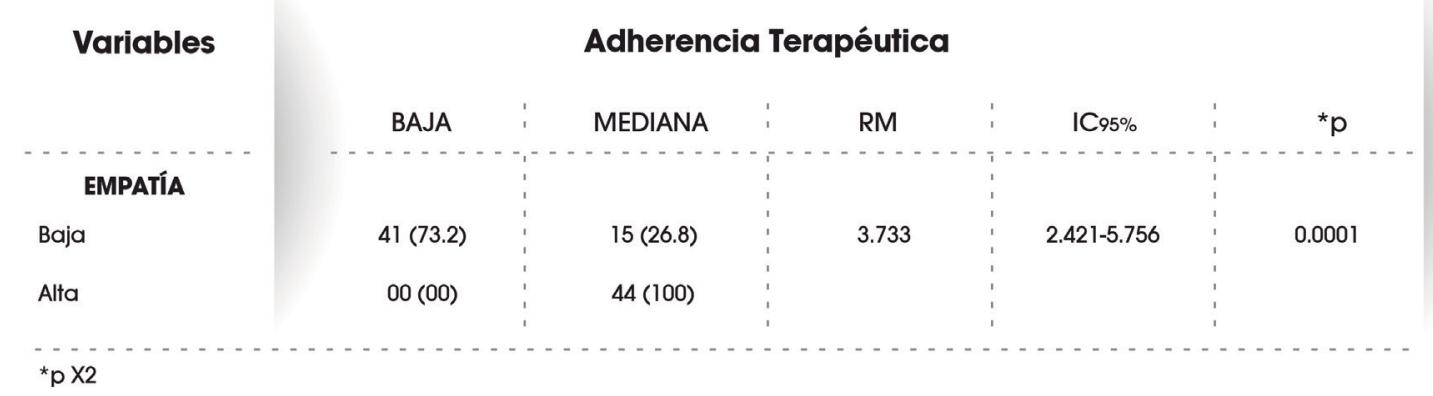

Los personas con empatía baja con el equipo de salud representan 2 veces más de riesgo que en los pacientes que conservan empatía alta, siendo una diferencia clínica y estadísticamente significativa, $(p<0.0001)$. 


\section{DISCUSIÓN}

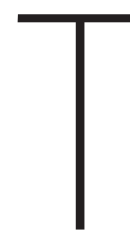

al como lo expresó Virginia Satir, el seno familiar es fundamental en la adherencia terapéutica, debido a que si la persona se encuentra interactuando con una familia conflictiva su autoestima se encontrara disminuida; lo que afectara la adherencia terapéutica, mientras los que habitan en un hogar nutricio su desenvolvimiento es diferente, armónico, existe comunicación y donde se fomenta la autoestima, tan indispensable en procesos de enfermedad crónica. Por lo que coincidimos con la teórica en este estudio que ha demostrado con resultados estadísticamente significativos que la autoestima a baja existe baja adherencia terapéutica.

Como lo ha demostrado el Instituto Nacional de Artritis y Enfermedades Musculo esqueléticas y de la Piel, en los Estados Unidos se demostró que el sexo femenino es el más afectado para la incidencia de la AR, nuestros resultados manifiestan que el 74\% de las personas estudiadas pertenecen al sexo femenino, además es de llamar la atención que su adherencia terapéutica es baja, situación que no había sido reflejada en otras investigaciones.

Sobre la comunicación del médico y la adhesión del paciente al tratamiento, se demostró que la comunicación médico está significativamente correlacionada positivamente con la adherencia de los pacientes; hay un 19\% más de riesgo de la falta de adherencia entre los pacientes cuyo médico comunica mal, que entre los pacientes que reciben una comunicación adecuada por su médico tratante. Nuestro estudio demostró que no existe empatía con el médico tratante en la consulta externa, lo cual incrementa el riesgo de AT.

\section{CONCLUSIONES}

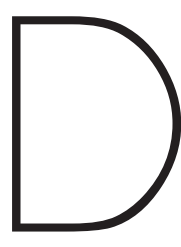
esde la postura teórica de Virginia Satir, se manifiesta que la familia es un recurso fundamental para mantener la adherencia terapéutica, y dentro del seno de una familia nutricia se favorece aún más, por lo que se comprueba la hipótesis de trabajo que a la AT está estrechamente relacionada con la funcionalidad familiar.

No existe una solución universal para mejorar la adherencia terapéutica, ya que influyen muchos factores. Lo ideal sería una combinación de esfuerzos en el seno de equipos multidisciplinarios, para obtener sinergias que multipliquen el resultado. Considerando que es vital la individualización para cada paciente.

Es de señalar que esta investigación se llevó a cabo en el ISSSTE Zaragoza, en donde el tratamiento se le proporciona al paciente, sin embargo es de interés explorar el comportamiento de este fenómeno en instituciones donde se atiende población abierta y que carecen de recursos económicos para adquirir los medicamentos, posiblemente esta condición afectará en el control de la Artritis Reumatoide.

Para la profesión de enfermería el cuidado tiene una importancia primordial. En las últimas décadas se ha identificado como el paradigma único a la disciplina y como "el corazón" de nuestra profesión, por lo que ella debe redireccionar su actuar retomando al seno familiar como un elemento fundamental en la adherencia terapéutica, esto sugiere la necesidad de nuevos modelos de atención primaria que deben ser abordados desde la formación del profesional de enfermería.

\section{6}

Vol. 14. No. 3 septiembre - diciembre 2015 
Considerando que existe un número reducido de médicos especialistas en reumatología, los profesionales de enfermería deben verlo como un lecho de oportunidad, en virtud de coadyuvar a enfrentar el proceso de la enfermedad, enlazar el apoyo con los integrantes de la familia, planificar un plan de alta de enfermería que permita un seguimiento del caso y participar en la formación del cuidador primario en el hogar, ello le permitirá reconocimiento social.

Finalmente contemplamos la posibilidad de realizar un estudio con un mayor número de pacientes y analizar otras variables que nos permitan dar una descripción más profunda de la falta de adherencia terapéutica.

\section{REFERENCIAS BIBLIOGRÁFICAS}

1. Sabaté E. Adherencia a los tratamientos de largo plazo: Pruebas para la acción. Organización Mundial de la Salud (OMS), 2004. Traducción de la Organización Panamericana de la Salud (OPS)

2. Ferrer $V$. Adherencia o cumplimiento de las prescripciones terapéuticas. Conceptos y factores implicados. Revista de Psicología Salud, 2000; 7 (1): 35-61.

3. OMS. La OMS alerta del bajo seguimiento de los tratamientos por parte de pacientes con enfermedades crónicas. Madrid. Ediciones Doyma. 2003.

4. Gertler P. The impact of Disease Management on Heatl, CPG Compliance, Quality, and Cost of Care Simcoe DRAFT. 2003; (12): 1-29

5. Colliere MF. Encontrar el sentido original de los cuidados enfermeros. Rev Enferm. 1999; 22(1):27-31.

6. Fausett, H.J. (2004). Artritis. En C.A. Warleld y H.J. Fausett (Eds.), Diagnóstico y tratamiento del dolor (p. 147-153). Barcelona: Masson

7. Guedes, C., Sumont-Fischer, D., Leichter-Nakache, S. y Boissier, M.C. (1999). Mortality in rheumatoid arthritis. Revue du Rhumatisme (England Edition), 66, 492-498.
8. OMS. Estrategias para mejorar la adherencia terapéutica en patologías crónicas. Información Terapéutica del Sistema Nacional de Salud. 2005. Disponible en: http://www.msc.es/biblioPublic/publicaciones/docs/vol29_2EstrategiasMejora.pdf. Consultado 17 mayo 2013.

9. Silva GE, Galeano E, Correa JO. Adherencia al tratamiento. Acta MED. Colomb 2005; 30:268-73.

10. Virginia Satir, Nuevas relaciones humanas en el núcleo familiar,ed. Pax México, 2areimpresión, 2005

11. Satir Virginia. キPsicoterapia Familiar Conjunta. Edit. La Prensa Médica Mexicana, México 1980.pag.41-50

12. Wittezaele Jean Jacques y Teresa García, La Escuela de Palo Alto. Historia y Evolución de las ideas esenciales. Barcelona, ed. Heder, 1994.

13. Gene Hunder, M.D. Hablemos claro sobre la artritis. Clínica Mayo. 2010 ed. Intersistemas. ISBN 978-607-443-034-9. pag. 49-60

14. Arraras, J.I. y Garrido, E. (2008). El afrontamiento de la enfermedad. Medicina y Salud (mayo-junio), 11. Disponible en red:http://www. zonahospitalaria.com.

Vol. 14. No. 3 septiembre - diciembre 2015 